\title{
Mitotic catastrophe and cell death induced by depletion of centrosomal proteins
}

\author{
M Kimura*,1, T Yoshioka ${ }^{1}$, M Saio ${ }^{2,4}$, Y Banno ${ }^{3}$, H Nagaoka ${ }^{1}$ and Y Okano ${ }^{1,5}$
}

Mitotic catastrophe, which refers to cell death or its prologue triggered by aberrant mitosis, can be induced by a heterogeneous group of stimuli, including chromosome damage or perturbation of the mitotic apparatus. We investigated the mechanism of mitotic catastrophe and cell death induced by depletion of centrosomal proteins that perturbs microtubule organization. We transfected cells harboring wild-type or mutated p53 with siRNAs targeting Aurora A, ninein, TOG, TACC3, $\gamma$-tubulin, or pericentriolar material-1, and monitored the effects on cell death. Knockdown of Aurora A, ninein, TOG, and TACC3 led to cell death, regardless of p53 status. Knockdown of Aurora A, ninein, and TOG, led to aberrant spindle formation and subsequent cell death, which was accompanied by several features of apoptosis, including nuclear condensation and Annexin V binding in HeLa cells. During this process, cleavage of poly(ADP-ribose) polymerase-1, caspase-3, and caspase-9 was detected, but cleavage of caspase-8 was not. Cell death, monitored by time-lapse imaging, occurred during both interphase and $M$ phase. In cells depleted of a centrosomal protein (Aurora A, ninein, or TOG), the rate of cell death was higher if the cells were cotransfected with siRNA against BubR1 or Mad2 than if they were transfected with siRNA against Bub1 or a control siRNA. These results suggest that metaphase arrest is necessary for the mitotic catastrophe and cell death caused by depletion of centrosomal proteins. Knockdown of centrosomal proteins led to increased phosphorylation of Chk2. Enhanced p-Chk2 localization was also observed at the centrosome in cells arrested in M phase, as well as in the nuclei of dying cells. Cotransfection of siRNAs against Chk2, in combination with depletion of a centrosomal protein, decreased the amount of cell death. Thus, Chk2 activity is indispensable for apoptosis after mitotic catastrophe induced by depletion of centrosomal proteins that perturbs microtubule organization.

Cell Death and Disease (2013) 4, e603; doi:10.1038/cddis.2013.108; published online 18 April 2013

Subject Category: Cancer

The term 'mitotic catastrophe' refers either to cell death or a moribund precursor state that is triggered by aberrant mitosis. Mitotic catastrophe can occur either during or after mitosis. ${ }^{1}$ Mitotic catastrophe can be induced by a heterogeneous group of stimuli, including chromosome damage and perturbation of the mitotic apparatus. When cells detect DNA damage, they arrest the cell cycle and may undergo apoptosis or senescence. However, when cells cannot maintain prolonged cell cycle arrest in G2 phase, they enter mitosis before DNA repair can be finished. This premature onset of mitosis leads to mitotic catastrophe and apoptosis. ${ }^{2}$ Reagents that affect spindle organization, including Aurora inhibitor and microtubule-targeting agents, also trigger mitotic catastrophe by disrupting the organization of the mitotic spindle. $^{3,4}$ In the case of mitotic catastrophe induced by cell fusion or DNA damage, caspases-2, -3 , and -9 are activated, ${ }^{5,6}$ but mitotic catastrophe can also cause caspase-independent apoptosis by activation of the spindle checkpoint in Bub1-deficient cells. ${ }^{7}$ In addition, multiple proapoptotic BCL-2 family members are involved in the execution of mitotic catastrophe and subsequent apoptosis by inhibition of Aurora A or Chk1. ${ }^{8,9}$

Microtubule organization is essential for many cellular processes, including intracellular transport, modulation of cell morphology and locomotion, and formation of the mitotic spindle. The centrosome, which is responsible for the nucleation of microtubules, consists of a pair of centrioles surrounded by pericentriolar material (PCM). Microtubule nucleation is mediated by $\gamma$-tubulin and additional subunits named $\gamma$-complex proteins (GCPs), which are localized in the PCM. ${ }^{10}$ Ninein, a coiled-coil protein localized at appendages/ satellites of centrioles, interact with GCPs and regulate $\gamma$-tubulin localization and microtubule nucleation. Pericentriolar matrix protein PCM-1 affects microtubule organization and centrosomal localization of ninein. ${ }^{11,12}$ The Aurora A, TACC3, and TOG proteins are localized to the PCM only during $M$ phase. $^{13-15}$ These proteins form a complex that regulates mitotic spindle organization. ${ }^{16}$

\footnotetext{
${ }^{1}$ Department of Molecular Pathobiochemistry, Gifu University Graduate School of Medicine, 1-1 Yanagido, Gifu, Japan; ${ }^{2}$ Department of Immunopathology, Gifu University Graduate School of Medicine, 1-1 Yanagido, Gifu, Japan; ${ }^{3}$ Department of Dermatology, Gifu University Graduate School of Medicine, 1-1 Yanagido, Gifu, Japan; ${ }^{4}$ Current address: Department of Pathology, University Hospital of the Ryukyus, 207 Uehara, Nishihara, Okinawa 903-0215, Japan. and ${ }^{5}$ Current address: Gifu University, 1-1 Yanagido, Gifu 501-1193, Japan.

${ }^{*}$ Corresponding author: M Kimura, Department of Molecular Pathobiochemistry, Gifu University Graduate School of Medicine, 1-1 Yanagido, Gifu 501 1194, Japan. Tel: +8158 230 6208; Fax: +81 58230 6209; E-mail: yo@ gifu-u.ac.jp

Keywords: centrosome; checkpoint; apoptosis; mitotic catastrophe; p53; caspase

Abbreviations: DAPI, 4', 6-diamidino-2-phenylindole; FBS, fetal bovine serum; GCP, $\gamma$-complex protein; PBS, phosphate-buffered saline; PCM, pericentriolar material; $\mathrm{PI}$, propidium iodide; SAC, spindle assembly checkpoint

Received 14.12.12; revised 02.2.13; accepted 07.2.13; Edited by G Raschellá
} 
Only a few studies have investigated the mechanisms underlying cell death in response to abnormalities in centrosomal proteins that perturbs microtubule organization. In acute myelogenous leukemia cell lines, the Aurora kinase inhibitor VX-680 induces cell death accompanied by formation of aberrant mitotic spindles and M-phase arrest. ${ }^{8}$ This effect is also observed in cells treated with siRNA against Aurora A. ${ }^{17}$ VX-680 decreases phosphorylation of Akt-1 and increases proteolytic cleavage of procaspase-3 and poly(ADP-ribose) polymerase-1 (PARP-1). VX-680 also increased the Bax/Bcl-2 protein ratio, a favorable proapoptotic predictor for survival. ${ }^{8}$ TACC3 knockdown also leads to mitotic arrest and cell death, which is accompanied by apoptotic features, such as Annexin $\mathrm{V}$ binding and caspase- 3 activation. ${ }^{18}$ However, the induction of mitotic catastrophe and subsequent cell death by inhibition of centrosomal proteins has not been investigated in detail. In addition, the differences between mitotic catastrophe induced by DNA damage or aberrant spindle formation have not been well studied.

In this study, we sought to determine the mechanism underlying mitotic catastrophe induced by depletion of centrosomal proteins that perturbs microtubule organization. Knockdown of centrosomal proteins, including Aurora A, ninein, TOG, and TACC3, led to high rates of aberrant spindle formation and subsequent cell death. Spindle assembly checkpoint (SAC) proteins and Chk2 were required for the induced mitotic catastrophe and subsequent cell death induced by depletion of Aurora A, ninein, or TOG.

\section{Results}

Cell cycle-dependent localization of centrosomal proteins. First, we examined the subcellular localization of six centrosomal proteins. To this end, we stained asynchronously growing HeLa cells with specific antibodies, and monitored the subcellular localization of the proteins in interphase and $\mathrm{M}$ phase. At the centrosome, we detected a strong fluorescence signal for Aurora A during $M$ phase, but only a very faint signal during interphase (Figure 1). In contrast, the centrosomal intensity of ninein was strong in interphase and weak in $M$ phase, almost disappearing by metaphase. TOG and TACC3 localized at the centrosome only during $\mathrm{M}$ phase. $\gamma$-Tubulin localized at the centrosome during both $\mathrm{M}$ phase and interphase, but more strongly during $\mathrm{M}$ phase. PCM-1 localized near the centrosome only during interphase.

\footnotetext{
Aberrant spindle formation and cell cycle arrest upon knockdown of centrosomal proteins. To investigate the function(s) of centrosomal proteins in mitosis and cell death, we transfected four cancer cell lines with siRNAs against the aforementioned centrosomal proteins. To confirm the knockdowns, we monitored protein levels by western blotting. The protein levels of Aurora A, ninein, TOG, TACC3, $\gamma$-tubulin, and $\mathrm{PCM}-1$ were decreased in cells transfected with the corresponding siRNAs, relative to the levels in control cells transfected with siRNA against GL3 (Figure 2a). To investigate the effect of these knockdowns on cell cycle progression, we monitored cell cycle distribution by flow cytometry. The proportion of cells in G2/M phase increased
}

upon knockdown of Aurora A, TOG, ninein, or TACC3, suggesting that these cells were arrested in G2/M phase (Figure 2b). In contrast, knockdown of either $\gamma$-tubulin or PCM-1 had no effect on cell cycle distribution. Next we examined the mitotic spindles of knockdown cells by immunofluorescence microscopy. We transfected HeLa cells with siRNA against Aurora A, ninein, TOG, or TACC3, and stained them with anti- $\alpha$-tubulin antibody and $4^{\prime}, 6$-diamidino2-phenylindole (DAPI). The morphology of the mitotic spindle was aberrant in these cells (Figures $2 \mathrm{c}$ and $\mathrm{d}$ ); the aberrant spindles included monopolar, asymmetrical, small, tripolar, or tetrapolar morphologies. Depletion of Aurora A, TOG, or TACC3 arrested the cells at late prometaphase or metaphase, but depletion of ninein arrested the cells at prometaphase, as determined by cell morphology and chromosome distribution. siRNA targeting $\gamma$-tubulin had little effect on spindle morphology, and siRNAs against PCM-1 and GL3 had no effect.

Apoptotic cell death upon knockdown of centrosomal proteins. Next we asked whether cells die after mitotic catastrophe following knockdown of centrosomal proteins. To address this question, we transfected p53-inactivated HeLa cell line, p53-mutated DLD-1 cell line, and two cell lines with functional p53 (HCT116 and U2OS), with siRNAs against the six aforementioned centrosomal proteins. After $72 \mathrm{~h}$ of transfection, we stained the cells with Hoechst 33342 and propidium iodide (PI). The percentage of PI-positive cells increased significantly following transfection with siRNAs targeting Aurora A, ninein, TOG, or TACC3 (Figures 3a and b). In addition, these cells exhibited nuclear condensation. siRNAs targeting $\gamma$-tubulin and PCM-1 induced slightly higher rates of cell death than the control siRNA. Cell death induced by siRNAs against Aurora A, ninein, TOG, or TACC3 was observed in all four of the cancer cells studied, suggesting that the observed cell death is independent of the p53 pathway.

For further analyses, we chose three proteins (Aurora A, ninein, and TOG) whose individual depletion induces cell death with high efficiency. We did not analyze the effects of $\gamma$-tubulin depletion further, because it did not induce cell death efficiently. We also did not analyze the effects of TACC3 knockdown, because induction of apoptosis by siRNA targeting TACC3 has been reported before. ${ }^{18}$ Next we asked whether this cell death is accompanied by apoptotic features. To this end, we monitored the percentage of Annexin V-positive cells. Forty-eight hours after transfection, we stained siRNA-transfected HeLa cells with FITC-labeled Annexin V, Hoechst 33342, and PI. The percentage of Annexin V-positive and Annexin V/PI double-positive cells increased following transfection with siRNAs targeting Aurora A, ninein, or TOG (Figures $3 c$ and $d$ ).

To elucidate the pathway underlying this type of cell death, we investigated caspase activity in the siRNA-transfected cells. Cell death induced by siRNAs targeting Aurora A, ninein, or TOG was accompanied by cleavage and activation of caspase-3. Caspase- 9 was also activated by these siRNAs, but cleavage of caspase-8 was not detected (Figure 3e). Partial digestion of PARP-1 was also detected in cells transfected with these three siRNAs. As the observed cell 
M phase

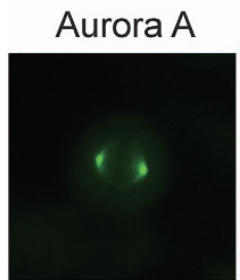

Ninein

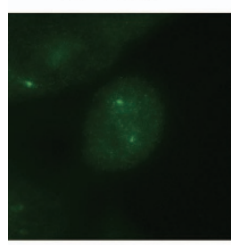

TOG

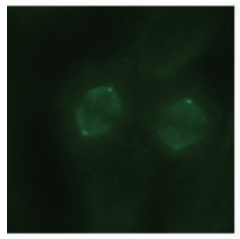

TACC3
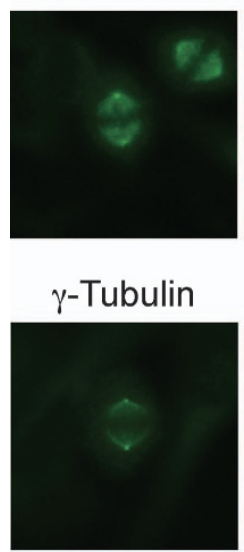

PCM-1

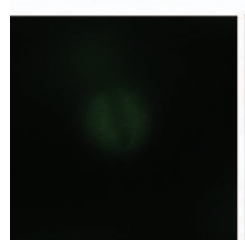

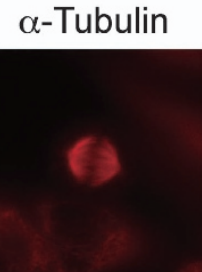

$\alpha$-Tubulin

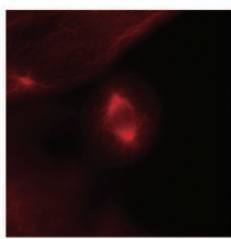

$\alpha$-Tubulin

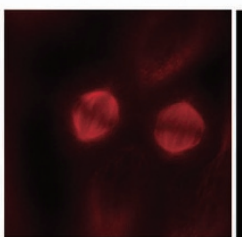

$\alpha$-Tubulin

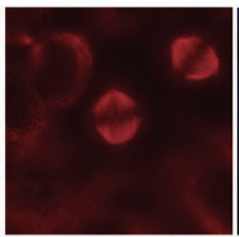

$\alpha$-Tubulin

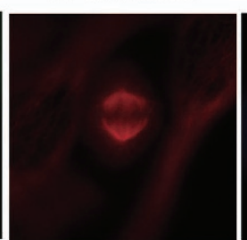

DNA

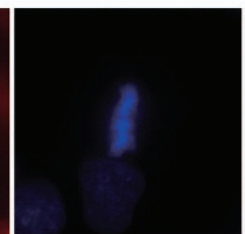

DNA

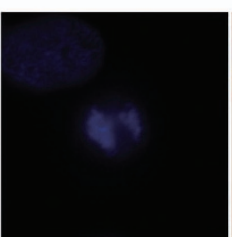

DNA

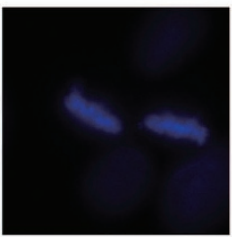

DNA

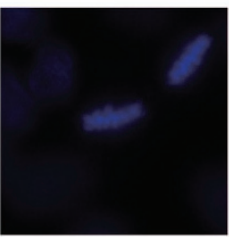

DNA

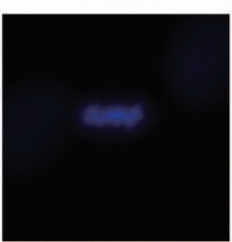

$\alpha$-Tubulin

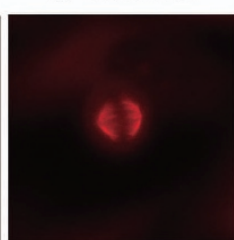

DNA

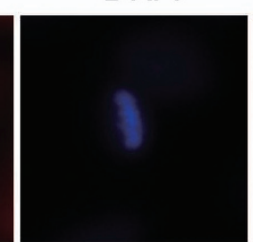

Merge

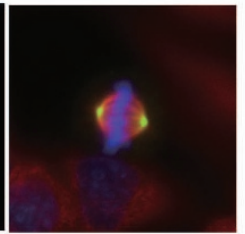

Merge

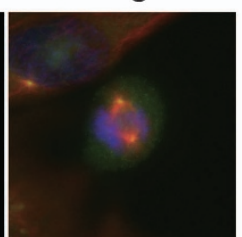

Merge

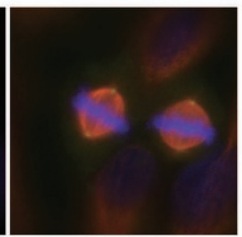

Merge

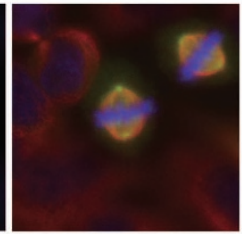

Merge

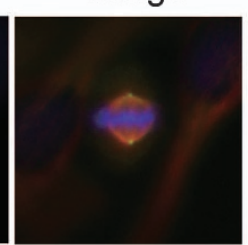

Merge

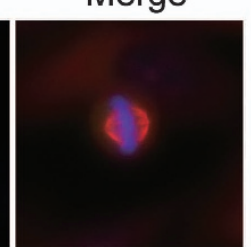

Interphase

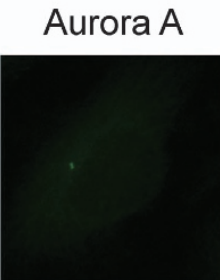

Ninein
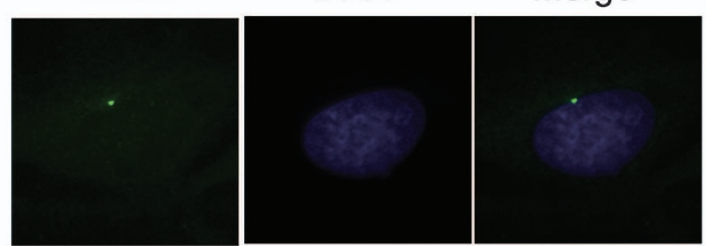

TOG

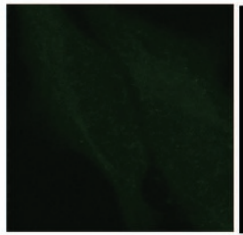

DNA

Merge

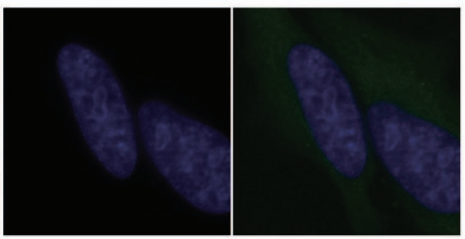

TACC3

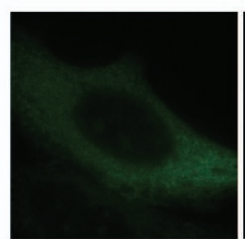

DNA

Merge
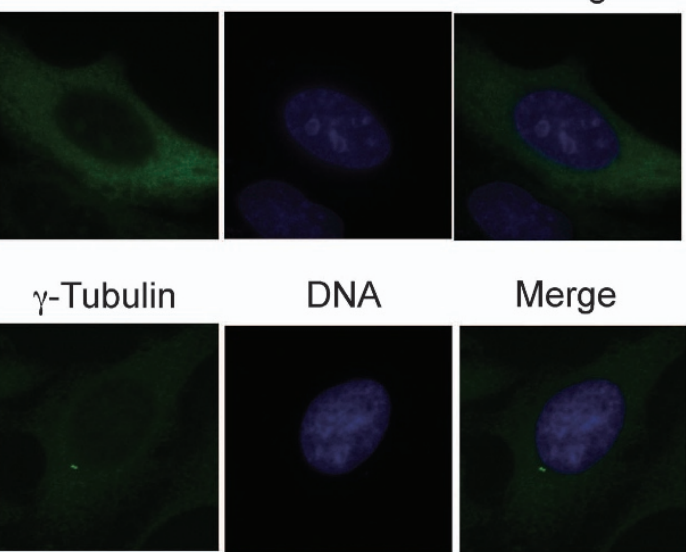

DNA

Merge
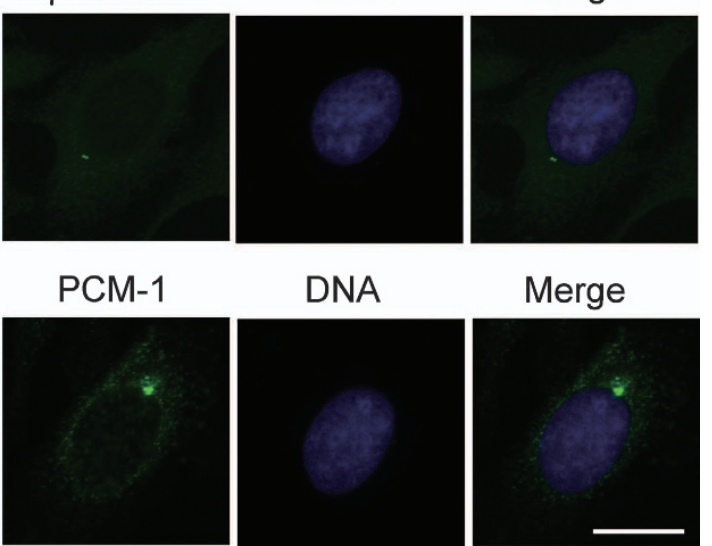

Figure 1 Subcellular localization of centrosomal proteins during M phase and interphase. Subcellular localization of the centrosomal proteins Aurora A, ninein, TOG, TACC3, $\gamma$-tubulin, and PCM-1 was examined by indirect immunofluorescence microscopy. HeLa cells were stained with polyclonal antibodies against the indicated centrosomal proteins, monoclonal anti- $\alpha$-tubulin antibody, and DAPI. Images of interphase and M phase cells are shown. Merged images are shown on the right. Bar, $20 \mu \mathrm{m}$

death was accompanied by Annexin $\mathrm{V}$ binding, nuclear condensation, and activation of caspases-3 and -9 , we conclude that cell death associated with mitotic catastrophe upon depletion of centrosomal proteins must be a type of apoptosis.
Apoptosis following mitotic catastrophe induced by knockdown of centrosomal proteins occurs in both $\mathbf{M}$ phase and interphase. To elucidate whether the cell death induced by depletion of centrosomal proteins occurs in $M$ phase or interphase, we analyzed time-lapse images of dying 
a

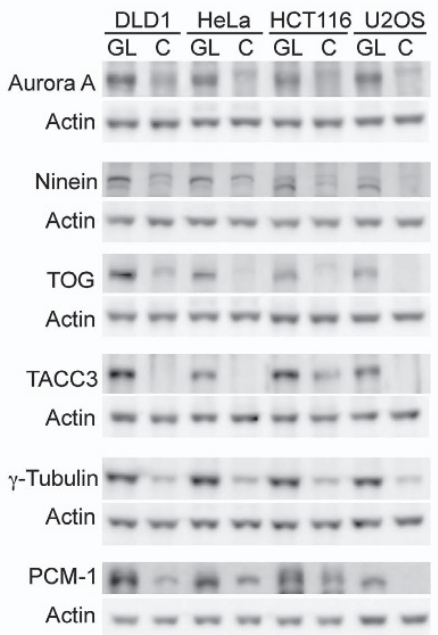

b
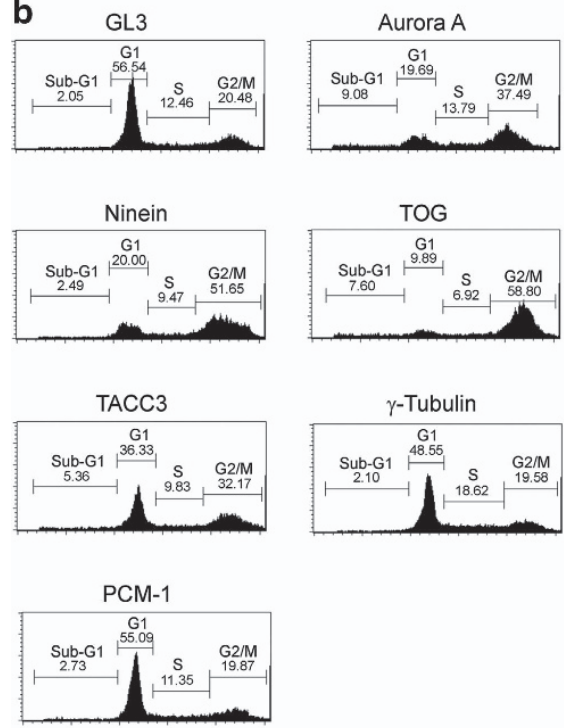

C
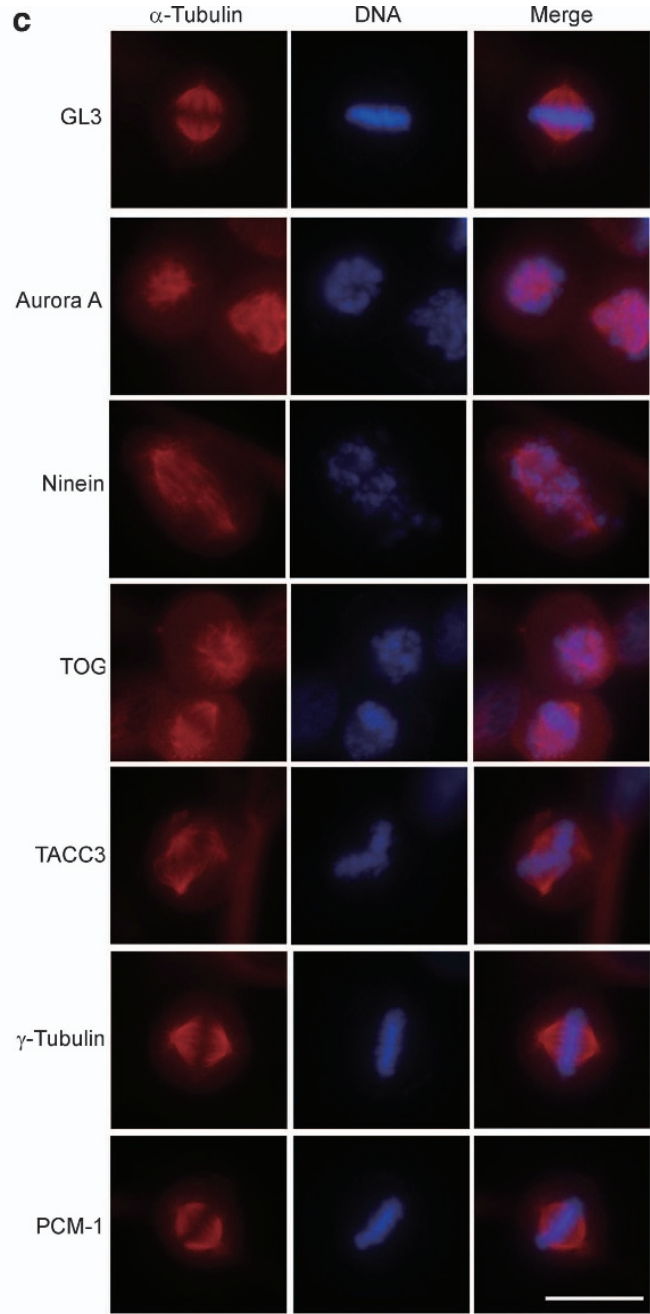

d

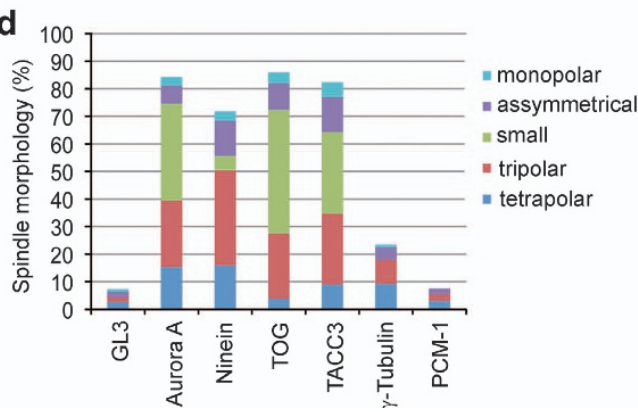

Figure 2 Aberrant spindle formation and cell cycle arrest induced by knockdown of centrosomal proteins. (a) The levels of centrosomal proteins in four cancer cell lines transfected with control siRNA (GL) or the indicated siRNAs targeting centrosomal proteins (C) were analyzed by western blotting $48 \mathrm{~h}$ after siRNA transfection. (b) HeLa cells $48 \mathrm{~h}$ after siRNA transfection were stained with $\mathrm{PI}$, and their nuclear DNA content was analyzed by flow cytometry. Percentages of cells in sub G1, G1, S, and G2/M phases are shown. Data shown were obtained from representative experiments, which were repeated three times with similar results. (c) HeLa cells $48 \mathrm{~h}$ after siRNA transfection were stained with anti- $\alpha$-tubulin antibody and DAPI. Bar, $20 \mu \mathrm{m}$. (d) Percentages of HeLa cells harboring aberrant spindles were counted in more than 100 randomly selected mitotic cells $48 \mathrm{~h}$ after siRNA transfection

cells. After $48 \mathrm{~h}$ of transfection, we collected images of siRNA-transfected HeLa cells at 3-min intervals for $3 \mathrm{~h}$. Cells transfected with siRNAs targeting Aurora A, ninein, or TOG died at a higher rate than control cells transfected with siRNA against GL3 (Figures $4 a$ and b). The stages at which cell death occurred were determined by cell morphology. Cells transfected with siRNA targeting Aurora A, ninein, or TOG arrested in $M$ phase and died during both $M$ phase and interphase (Supplementary Movies S1-S4). More TOGdepleted cells died during $M$ phase than during interphase. Conversely, more Aurora A- and ninein-depleted cells died during interphase than during $\mathrm{M}$ phase. Overall, these results 


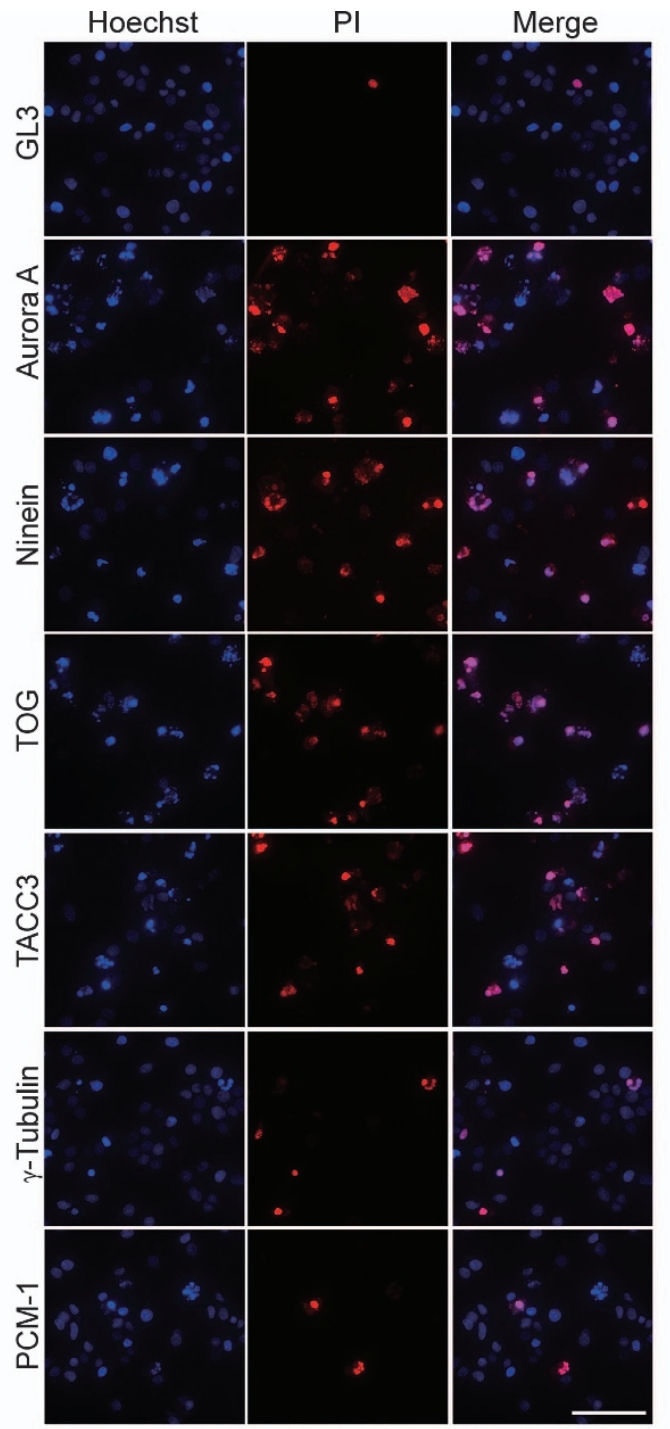

c

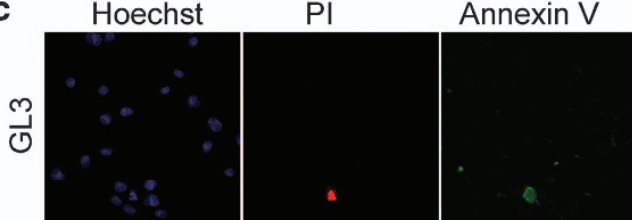

진
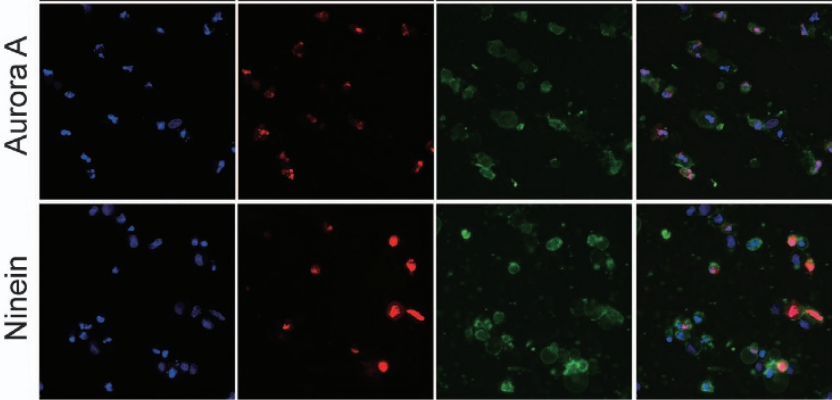

인

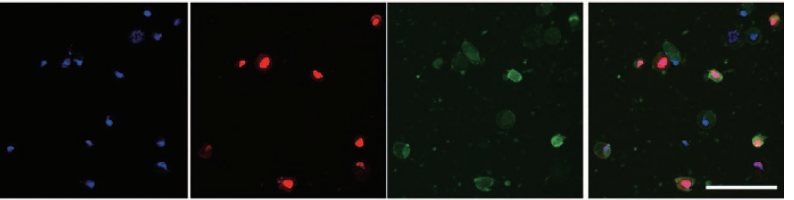

d

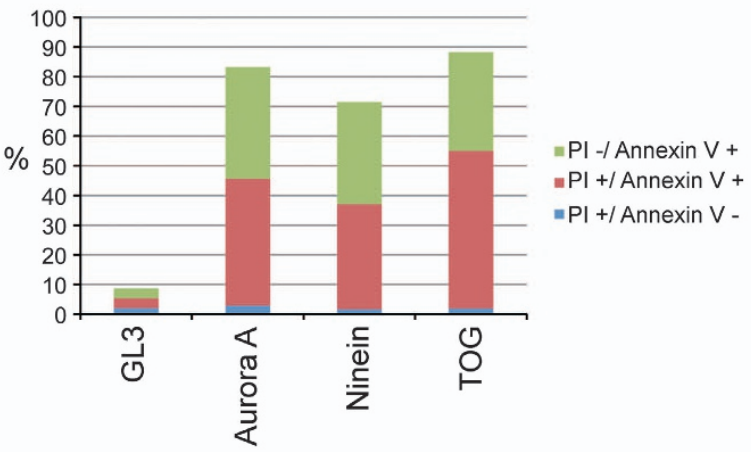

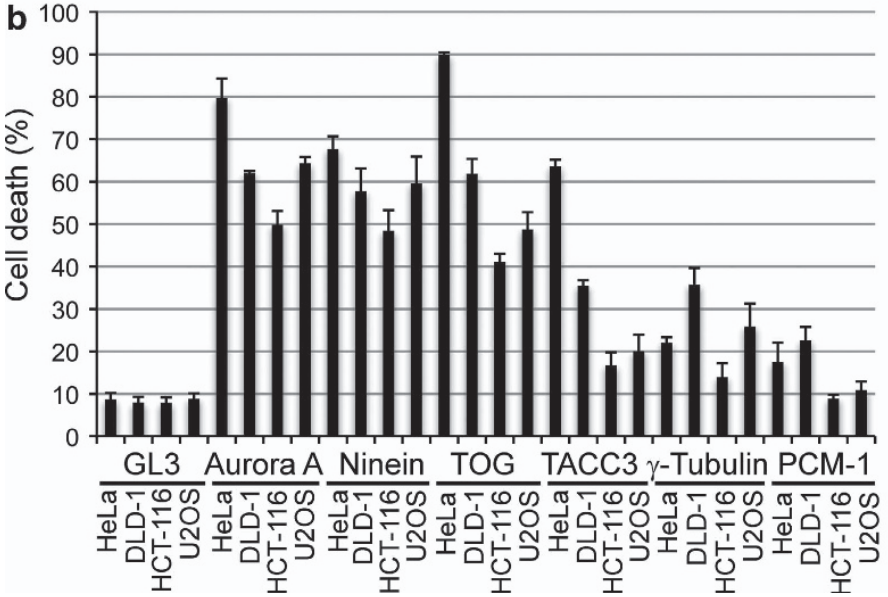

e

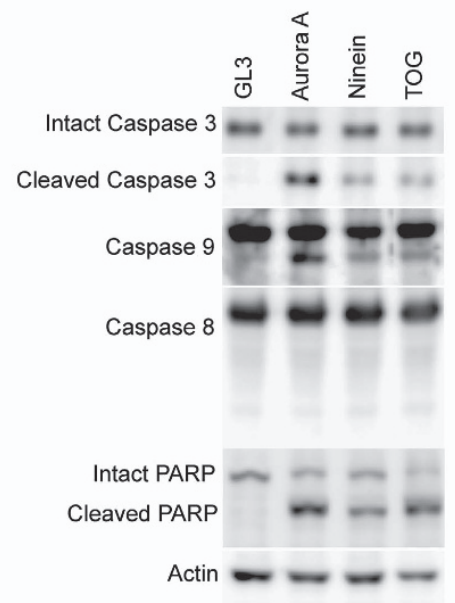

Figure 3 Cell death induced by knockdown of centrosomal proteins. (a) Nuclear morphology of HeLa cells $72 \mathrm{~h}$ after siRNA transfection. Nuclei of siRNA-transfected cells were stained with PI and Hoechst 33342. Bar, $100 \mu \mathrm{m}$. (b) Percentages of PI-positive cells in four cancer cell lines $72 \mathrm{~h}$ after siRNA transfection. More than 200 randomly selected cells were counted for each sample. Values are an average of three independent experiments. Error bars represent S.D. (c) HeLa cells $48 \mathrm{~h}$ after siRNA transfection were stained with FITC-labeled Annexin V, PI, and Hoechst 33342. Bar, $100 \mu \mathrm{m}$. (d) PI and Annexin V-positive HeLa cells $48 \mathrm{~h}$ after siRNA transfection. Over 200 randomly selected cells were counted. Values are an average of three independent experiments. Error bars represent S.D. (e) Caspases and PARP-1 were partially cleaved during cell death following mitotic catastrophe induced by knockdown of centrosomal proteins. Lysates from HeLa cells $48 \mathrm{~h}$ after siRNA transfection were analyzed by western blotting 
a
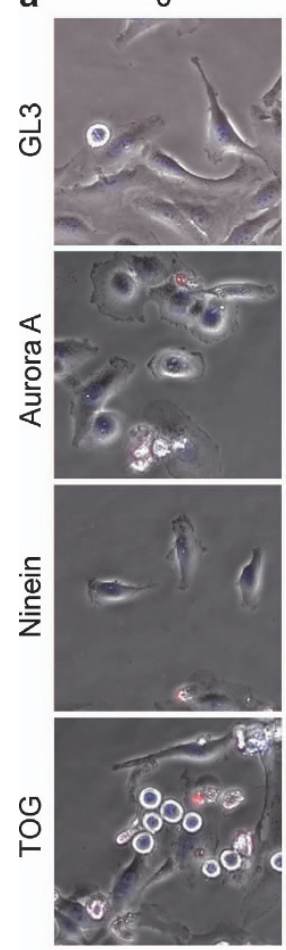

30

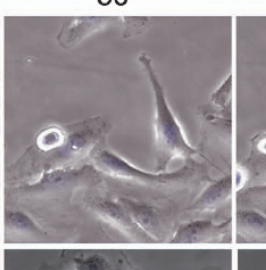

60
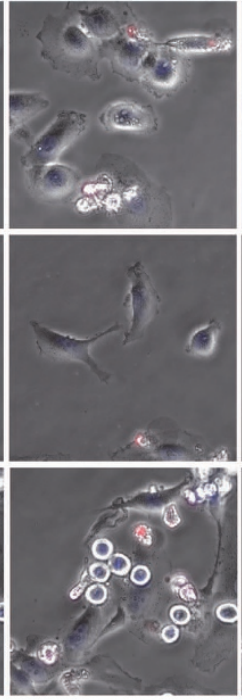
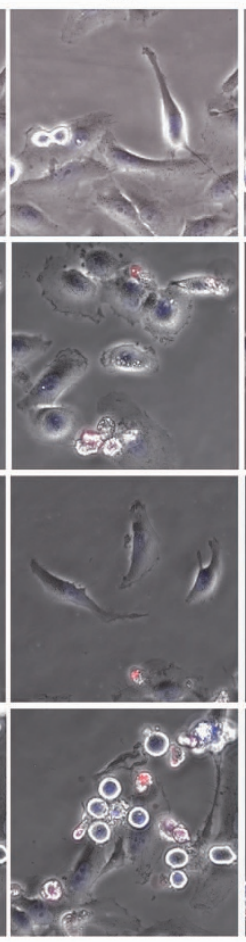

90
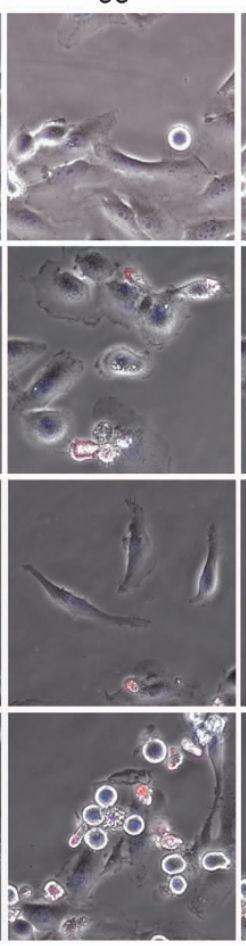

120
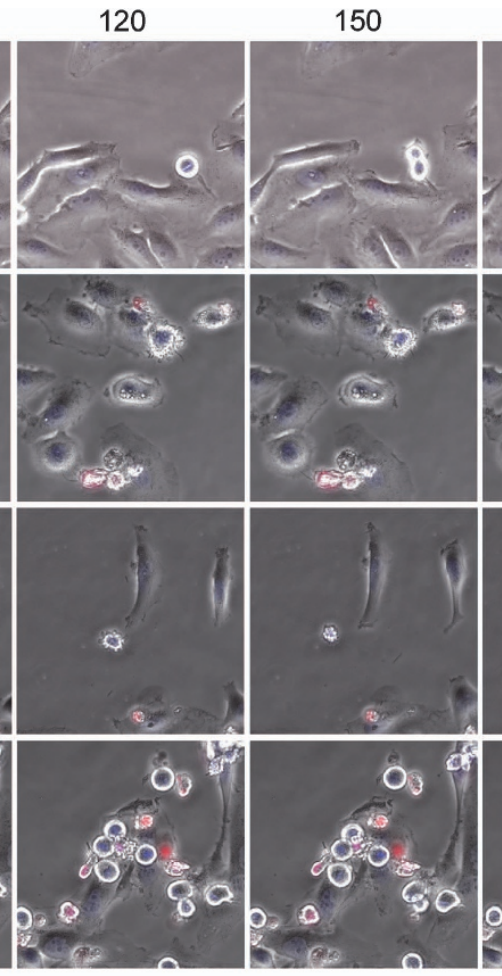

$180 \min$
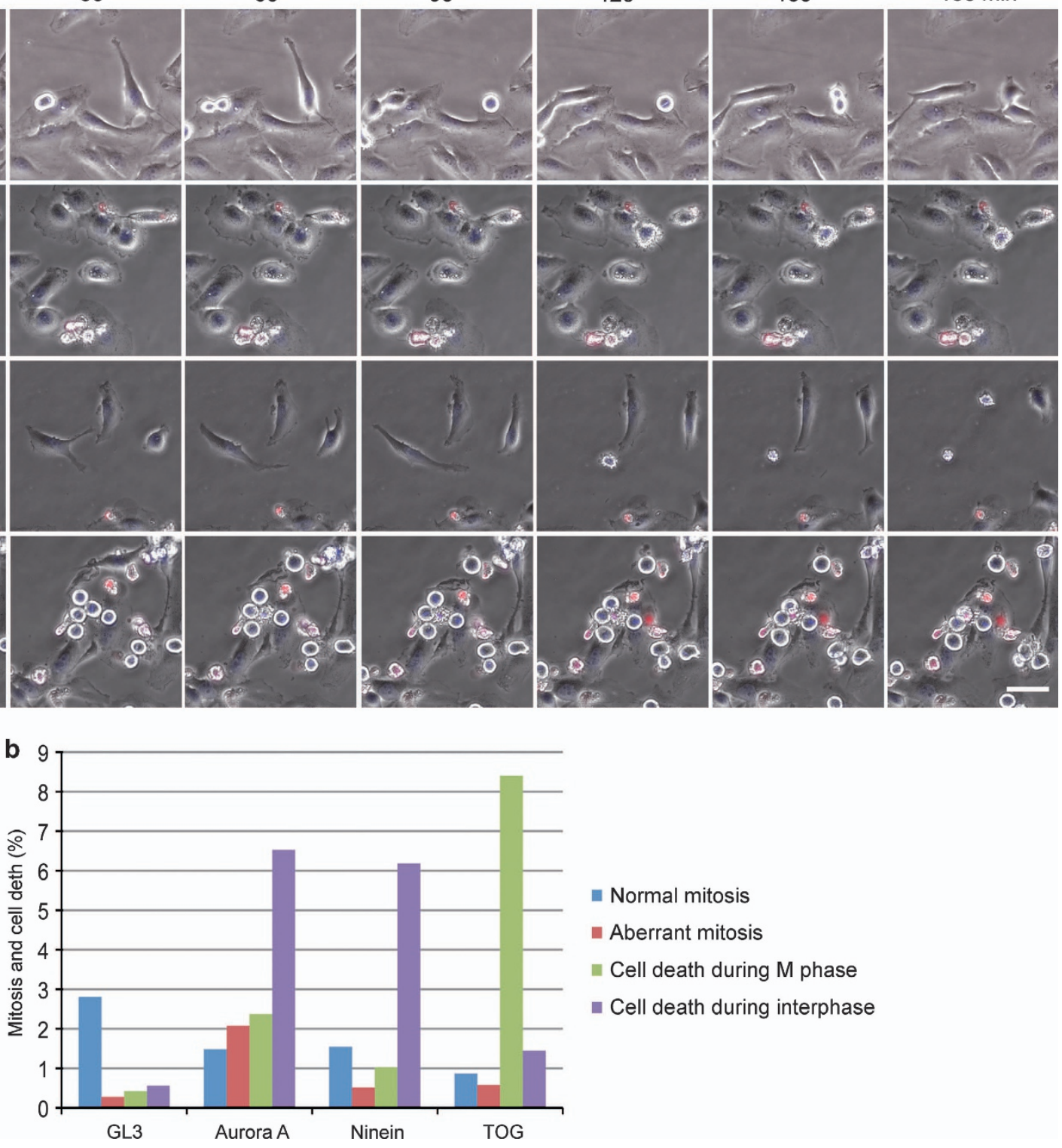

- Normal mitosis

- Aberrant mitosis

a Cell death during $\mathrm{M}$ phase

- Cell death during interphase

Figure 4 Apoptosis following mitotic catastrophe induced by knockdown of centrosomal proteins occurred in both mitotic phase and interphase. (a) Time-lapse imaging of HeLa cells depleted of centrosomal proteins. Cells were monitored from 48 to $51 \mathrm{~h}$ after transfection. Bar, $50 \mu \mathrm{m}$. (b) Percentages of cells that died in interphase or M phase. Over 300 randomly selected cells were counted for each sample

suggest that depletion of centrosomal proteins does not fate a cell to die at a specific stage of the cell cycle, but rather that cells can die during or after mitosis.

\section{Apoptosis induced by knockdown of centrosomal proteins depends on the SAC. To determine whether SAC has a role in cell death following mitotic catastrophe induced by centrosomal protein depletion, we studied the effects on cell viability of siRNAs against factors involved in the SAC. We transfected HeLa cells with siRNA targeting Bub1, BubR1, or Mad2, and confirmed the knockdowns by western blotting (Figure 5a). Next we transfected cells simultaneously with siRNAs targeting a centrosomal protein and a SAC component, and counted the cells arrested at}

M phase. Cells cotransfected with siRNAs against GL3 and one of the centrosomal proteins (Aurora A, ninein, or TOG) arrested at $M$ phase. Likewise, cells cotransfected with siRNAs against Bub1 and a centrosomal protein also arrested at $\mathrm{M}$ phase. However, cells transfected with siRNAs against either BubR1 or Mad2 and a centrosomal protein did not arrest at $M$ phase (Figures $5 b$ and $c$ ). We also investigated the rate of cell death under these conditions. In cells transfected with siRNAs against GL3 or Bub1 and a centrosomal protein, the death rate was high. In contrast, in cells transfected with siRNAs against either BubR1 or Mad2 and a centrosomal protein, the death rate was lower (Figure 5d). These results suggest that the metaphase arrest induced by the SAC is required for this type of cell death. 

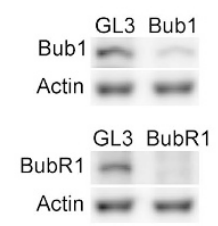

GL3 Mad2

Mad2 -

Actin $=$

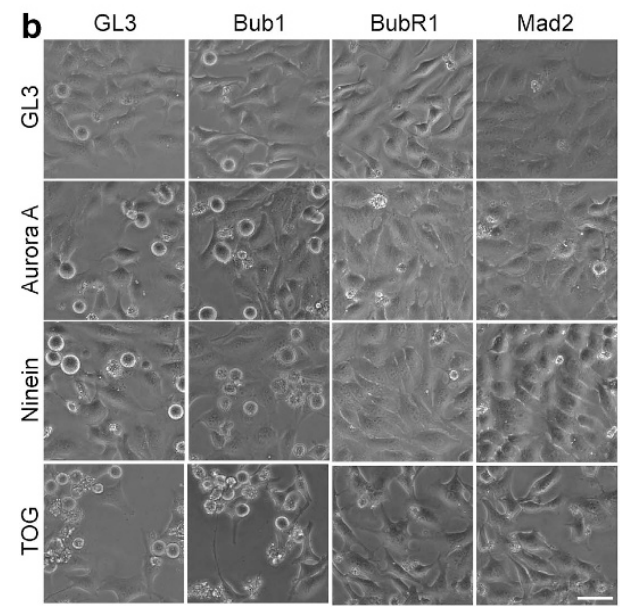

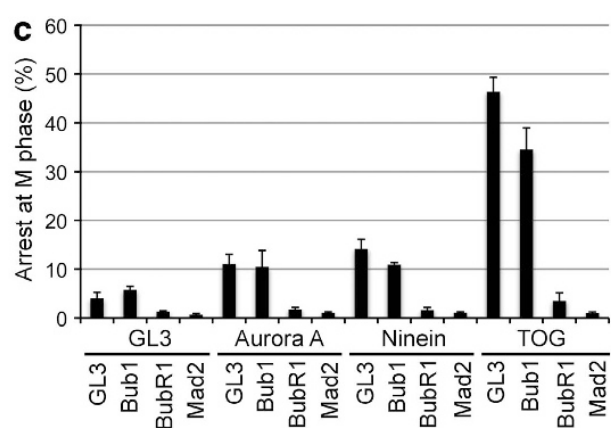

d

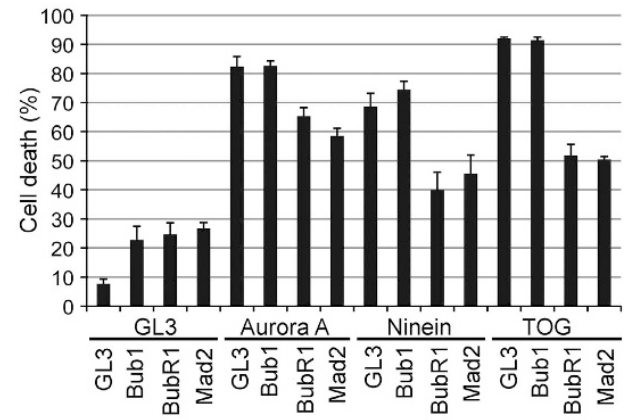

Figure 5 Apoptosis following mitotic catastrophe induced by knockdown of centrosomal proteins depends on the SAC. (a) The levels of Bub1, BubR1, and Mad2 proteins in HeLa cells transfected with the corresponding siRNAs were analyzed by western blotting $48 \mathrm{~h}$ after siRNA transfection. (b) siRNAs against SAC factors and centrosomal proteins were transfected simultaneously. Cell images were acquired using a phase-contrast microscope $48 \mathrm{~h}$ after siRNA transfection. Bar, $50 \mu \mathrm{m}$. (c) Percentages of arrested cells at M phase following cotransfection with siRNAs against centrosomal and SAC proteins $48 \mathrm{~h}$ after siRNA transfection. Over 200 randomly selected cells were counted for each sample. Values are an average of three independent experiments. Error bars represent S.D. (d) Rates of cell death (expressed as percentages) induced by knockdown of centrosomal and SAC proteins $48 \mathrm{~h}$ after siRNA transfection. Over 200 randomly selected cells were counted for each sample. Values are an average of three independent experiments. Error bars represent S.D.

Apoptosis induced by knockdown of centrosomal proteins depends on Chk2 activity. To investigate whether Chk2 has a role in cell death induced by depletion of centrosomal proteins that perturbs microtubule organization, we monitored the phosphorylation status of Chk2 at Thr-68, which is important for its activation. Phosphorylation of Chk2 was enhanced upon knockdown of either Aurora A, ninein, or TOG (Figure 6a). We then studied the subcellular localization of Chk2 and its phosphorylated form (p-Chk2) by immunofluorescence microscopy. Chk2 localized to the nucleus in interphase and to the centrosome in $\mathrm{M}$ phase. In damaged cells, $p$-Chk2 localized in a similar pattern to the nucleus of damaged cells during interphase, and to the centrosome during $\mathrm{M}$ phase. The $\mathrm{p}$-Chk2 signal was more intense at the centrosome of cells arrested in $\mathrm{M}$ phase and in the nuclei of a subpopulation of dying cells (Figure 6b).

Next we investigated the effect of Chk2 knockdown on cell death. We transfected HeLa cells with siRNA against Chk2 and confirmed the knockdown by western blotting (Figure 6c). We then simultaneously transfected cells with siRNAs targeting Chk2 and a centrosomal protein, and found that the rate of cell death was decreased by Chk2 siRNA (Figure 6d). These results suggest that phosphorylation of Chk2 occurs in the dying cells, and that its activity is required for subsequent apoptosis.

\section{Discussion}

The centrosome, which contains $\sim 100$ proteins, is responsible for the nucleation and organization of microtubules. We depleted cells of six centrosomal proteins and analyzed the effects on spindle morphology and cell death. Aurora A, TACC3, and TOG localized to the centrosome only during M phase (Figure 1). In contrast, the fluorescence intensity of ninein at the centrosome was strong in interphase and weak in $M$ phase, almost disappearing by metaphase. Knockdown of Aurora A, TOG, ninein, or TACC3 led to aberrant mitotic spindle formation, arrest in $\mathrm{G} 2 / \mathrm{M}$ phase, and cell death (Figures 2 and 7a). Localization of $\gamma$-tubulin at the centrosome was observed throughout the cell cycle, but a stronger signal was observed in M phase. PCM-1 localized to the centrosome only in interphase. Knockdown of $\gamma$-tubulin exhibited little effect, and knockdown of PCM-1 had no effect on spindle formation and cell cycle progression. These results suggest that a subset of centrosomal proteins directly regulate the mitotic spindle, and that depletion of these factors effectively induces a type of mitotic catastrophe.

p53 has a critical role in apoptosis induced by DNA damage. ${ }^{19}$ Although p73 has important roles in mitotic catastrophe induced by microtubule-targeting agents, p53 
a

a

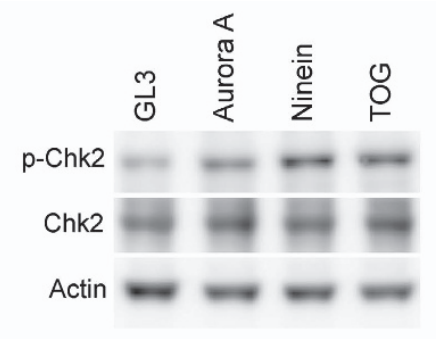

c

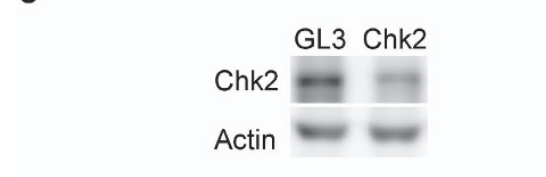

d

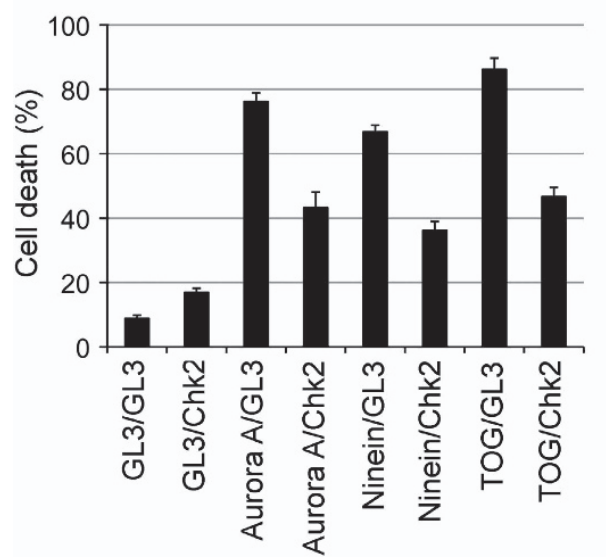

b
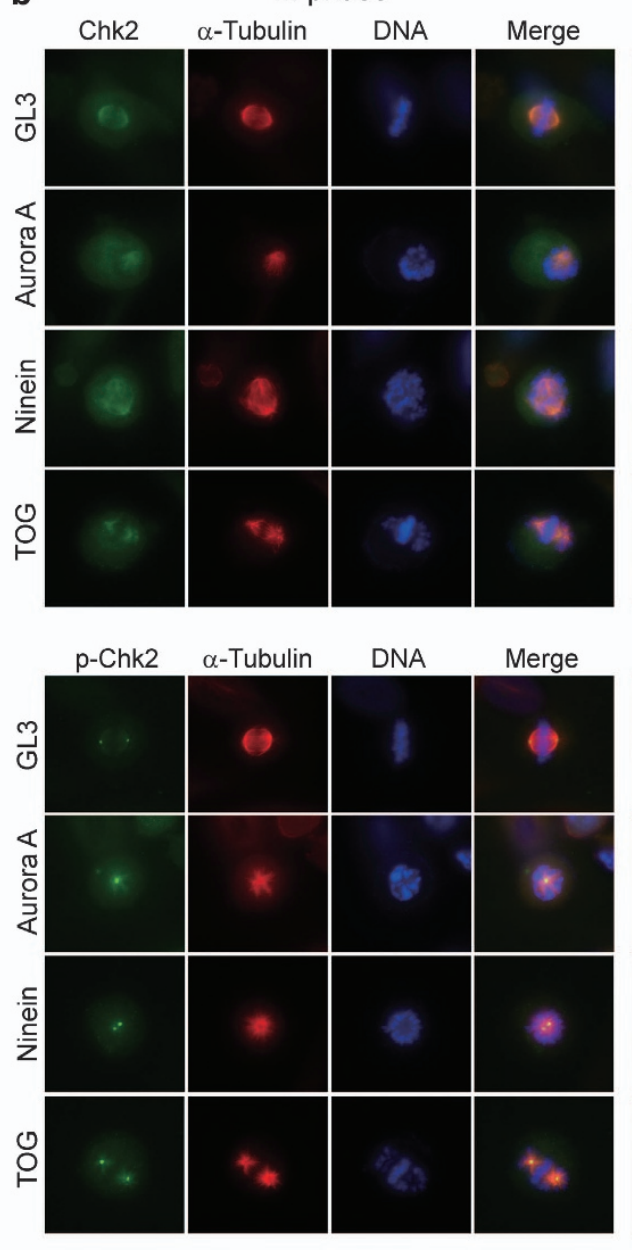

$M$ phase

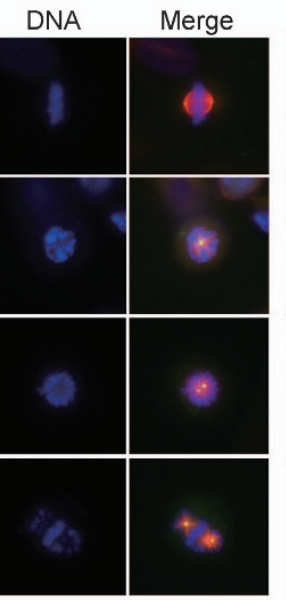

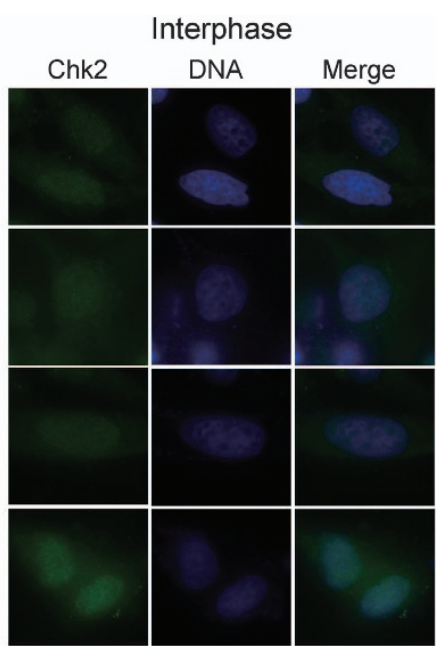

p-Chk2

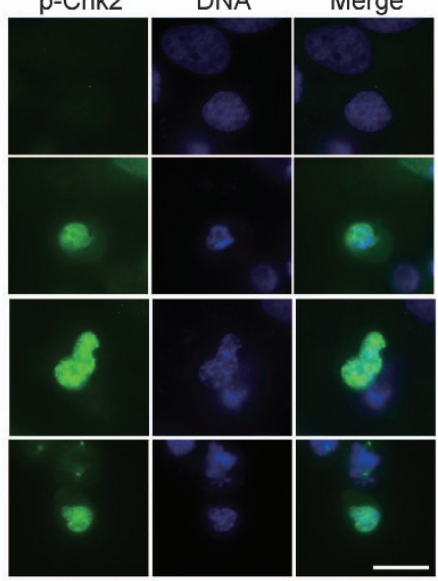

Figure 6 Apoptosis following mitotic catastrophe induced by knockdown of centrosomal proteins depends on Chk2. (a) Levels of Chk2 and its phosphorylated form (p-Chk2) in HeLa cells transfected with siRNA for centrosomal proteins. Lysates obtained $48 \mathrm{~h}$ after siRNA transfection were analyzed by western blotting. (b) Subcellular localization of Chk2 and p-Chk2 in siRNA-transfected cells $48 \mathrm{~h}$ after transfection. Bar, $20 \mu \mathrm{m}$. (c) The level of Chk2 in HeLa cells cotransfected with GL3 control siRNA and siRNA against Chk2 was analyzed by western blotting $48 \mathrm{~h}$ after siRNA transfection. (d) Rates of cell death (expressed as percentages) induced by simultaneous knockdown of centrosomal proteins and Chk2. At least 200 randomly selected cells were counted $48 \mathrm{~h}$ after siRNA transfection. Values are an average of three independent experiments. Error bars represent S.D.

does not. ${ }^{20,21}$ The results presented in this study suggest that p53 is also not essential for mitotic catastrophe and cell death induced by depletion of centrosomal proteins. In M phase, transcription is thought to be largely inactive. For this reason, and as centrosomal proteins directly regulate the mitotic spindle during $\mathrm{M}$ phase, cell death in $\mathrm{M}$ phase is probably not regulated by p53 transcriptional activation. In contrast, cell death after mitosis can be regulated by $\mathrm{p} 53$ transcriptional activation or by the p53-inducible protein p21.

Cell death induced by depletion of Aurora A, ninein, or TOG exhibited apoptotic features, such as Annexin $\mathrm{V}$ binding and nuclear condensation (Figure 3 ). In addition, cleavage of PARP-1 and caspases- 3 and -9 was detected, but cleavage of caspase-8 was not (Figure 3). In a previous study, depletion of TACC3 led to mitotic arrest and cell death, which was accompanied by apoptotic features, such as Annexin V binding and caspase-3 activation, ${ }^{18}$ and the Aurora kinase inhibitor VX-680 increased proteolytic cleavage of procaspase-3 and PARP $-1 .{ }^{8}$ These findings are consistent with our results regarding Aurora A knockdown.
The timing of cell death due to mitotic catastrophe induced by depletion of centrosomal proteins has not been investigated in detail. To address this point, we monitored knockdown cells using live-cell imaging, and observed that cell death following depletion of centrosomal proteins occurred in both interphase and $M$ phase. Knockdown of TOG induced apoptosis in $M$ phase rather than in interphase, whereas depletion of Aurora $A$ or ninein led to apoptosis in interphase rather than $\mathrm{M}$ phase (Figure 4). We conclude that the timing of death due to mitotic catastrophe induced by depletion of centrosomal proteins is not strictly determined and can occur either during or after mitosis.

To determine whether SAC has a role in cell death, we studied the effects of siRNA against factors involved in the SAC. When siRNAs against either BubR1 or Mad2 and a centrosomal protein (Aurora A, ninein, or TOG) were transfected in combination, the percentage of cells arrested in $\mathrm{M}$ phase and the rate of cell death were lower than in cells cotransfected with siRNAs against either GL3 or Bub1 and a centrosomal protein (Figure 5). These results suggest that metaphase arrest induced by the SAC is required for the mitotic 
catastrophe and subsequent cell death induced by depletion of centrosomal proteins (Figure 7b). Therefore, although spindle morphology or correct chromosome segregation are not important determinants of mitotic catastrophe and cell death induced by depletion of centrosomal proteins, prolonged arrest at $\mathrm{M}$ phase is crucial. Previous studies make conflicting claims about the dependency of mitotic catastrophe on specific SAC factors: one study reported that BubR1 and Mad2 are indispensable for mitotic catastrophe induced by inhibition of Chk $1,{ }^{9}$ whereas another reported that Mad2 is required for mitotic catastrophe induced by Glyfoline, which does not bind DNA and does not affect tubulin polymerization. ${ }^{22}$ Thus, there remains some controversy regarding the role of the SAC in mitotic catastrophe. As mitotic catastrophe can be induced by a variety of stimuli, there must be several signal transduction pathways that trigger mitotic catastrophe.

In DNA damage-induced apoptosis, Chk2 is activated by phosphorylation at Thr- $68 .{ }^{23} \mathrm{p}$-Chk2 localizes to nuclear foci at DNA damage sites or mitotic centrosome. ${ }^{24}$ In Drosophila, Chk2 has a role in signal transduction, leading to mitotic catastrophe induced by DNA replication inhibitors and DNA-damaging agents. $^{25}$ To determine whether Chk2 has a function in cell death following mitotic catastrophe induced by depletion of centrosomal proteins that perturbs microtubule organization, we simultaneously transfected cells with siRNAs against Chk2 and a centrosomal protein, and monitored the effect on the cell death. The death rate was decreased in cells transfected with Chk2 siRNA (Figure 6), suggesting that Chk2 is necessary for cell death after mitotic catastrophe induced by depletion of centrosomal proteins (Figure 7c). In addition, phosphorylation of Chk2 in the nucleus of dying cells increased, following knockdown of centrosomal proteins. These results suggest that phosphorylation of Chk2 at the centrosome in M phase or in the nucleus in interphase is enhanced in dying cells, and that its activity is necessary for apoptosis following mitotic catastrophe induced by depletion of centrosomal proteins. Thus, in addition to its function in the DNA-damage checkpoint in interphase, Chk2, which is localized at the centrosome in M phase, also has a role in mitotic catastrophe and cell death induced by depletion of centrosomal proteins that perturbs microtubule organization.

In conclusion, siRNA-mediated depletion of a subset of centrosomal proteins, including Aurora A, ninein, and TOG, leads to mitotic catastrophe and subsequent cell death. This type of cell death, induced by depletion of centrosomal proteins that perturbs microtubule organization, requires SAC proteins and Chk2. Mitotic catastrophe and subsequent apoptosis represent a novel antineoplastic mechanism that should be investigated in the context of cancer prevention and therapy.

\section{Materials and Methods}

Cell culture and transfection. HeLa (cervical carcinoma), U2OS (osteosarcoma), HCT116 (colorectal carcinoma), and DLD-1 (colorectal adenocarcinoma) cells were cultured in Dulbecco's modified Eagle medium supplemented with $10 \%$ fetal bovine serum (FBS). siRNAs were transfected into cells using RNAiMAX (Invitrogen, Carlsbad, CA, USA).

RNA interference was performed with synthesized double-stranded RNAs specific for human Aurora A (5'-AUGCCCUGUCUUACUGUCATT-3' $\left.{ }^{\prime}\right)$, ninein $\left(5^{\prime}\right.$-UAUGAGCAUUGAGGCAGAGTT- $\left.3^{\prime}\right),{ }^{27}$ TOG $\left(5^{\prime}\right.$-GAGCCCAGAGUGGUCC AAATT- $\left.3^{\prime}\right),{ }^{14}$ TACC3 (5'-GUUACCGGAAGAUCGUCUGTT-3' $\left.{ }^{\prime}\right),{ }^{28}$ PCM-1 (5'-UC AGCUUCGUGAUUCUCAGTT-3'), ${ }^{29} \gamma$-tubulin $\left(5^{\prime}\right.$-GGAGGACAUGUUCAAGGA ATT- $\left.3^{\prime}\right),{ }^{30}$ Bub1 (5'-AUACCACAAUGACCCAAGATT-3'), ${ }^{31}$ BubR1 (5'-CGGGCA a

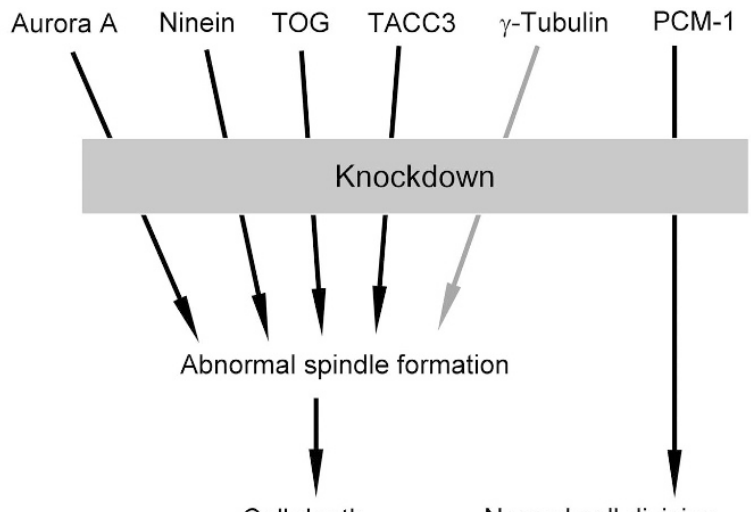

b

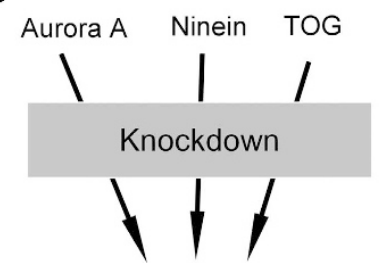

Spindle assembly checkpoint

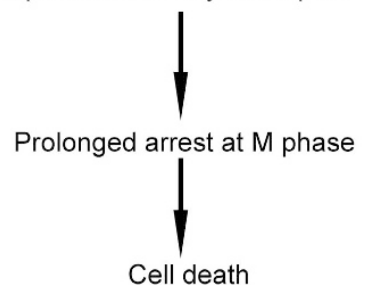

C

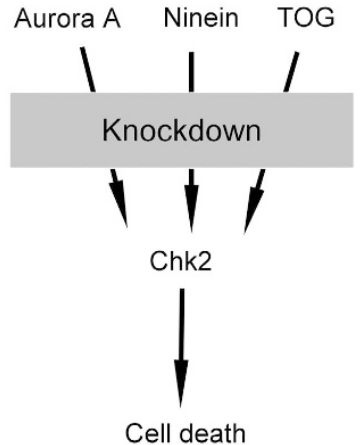

Figure 7 Hypothetical model explaining the pathway for apoptosis following centrosomal protein depletion. (a) Mitotic catastrophe is induced by knockdown of one of a subset of centrosomal proteins and followed by cell death. This type of cell death depends on the SAC (b) and Chk2 (c)

UUUGAAUAUGAAATT-3'), ${ }^{31}$ Mad2 (5'-ACCUUUACUCGAGUGCAGATT-3'), ${ }^{32}$ Chk2 (5'-GAACCUGAGGACCAAGAACTT-3'), ${ }^{33}$ or GL3 (used here as a negative control) (5'-CUUACGCUGAGUACUUCGATT-3'). ${ }^{33}$

Antibodies and chemicals. Polyclonal antisera against Aurora A/AlK were generated and purified as previously described. ${ }^{13}$ Antibodies against Bub1 and BubR1 were kindly provided by Taylor et al. ${ }^{34}$ Antibodies against $\alpha$-tubulin, actin, Chk2, p-Chk2 (Thr-68), and caspase-8 were obtained from Sigma (St. Louis, MO, USA). Antibodies against ninein, TOG, and $\gamma$-tubulin were purchased from BioLegend (San Diego, CA, USA). Antibodies against TACC3, intact caspases-3 and -9 , and PARP-1 were obtained from Santa Cruz Biotechnology (Santa Cruz, CA, USA). Antibodies against PCM-1 and Mad2 were purchased from Bethyl (Montgomery, TX, USA). Antibodies against cleaved caspase-3 were purchased from Trevigen (Helgerman, MD, USA). DAPI and PI were obtained from Wako (Osaka, Japan) and Sigma, respectively.

Immunofluorescence microscopy. To analyze nuclear morphology and monitor cell death, Hoechst 33342 was added to the culture medium for $1 \mathrm{~h}$, and then PI was added for an additional $15 \mathrm{~min}$. Cells were collected by centrifugation and washed with phosphate-buffered saline (PBS). To detect phosphatidylserine exposure, we used the Annexin V FLUOS staining kit (Roche, Basel, Switzerland). Fluorescence was observed using a BIOREVO BZ-9000 microscope (KEYENCE, Osaka, Japan).

For indirect immunofluorescence experiments, HeLa cells on coverslips were fixed with either methanol or paraformaldehyde. For methanol fixation, cells were washed with PBS and fixed with absolute methanol for 2 min and incubated with 
PBS for 10 min. For paraformaldehyde fixation, cells were washed with PBS and fixed with $4 \%$ paraformaldehyde for $10 \mathrm{~min}$. To permeabilize, fixed cells were incubated with $0.2 \%$ TritonX-100 in PBS for $10 \mathrm{~min}$, and then in $50 \mathrm{mM}$ glycine in PBS for $10 \mathrm{~min}$ at room temperature. Fixed and permeabilized cells were blocked with $0.5 \%$ bovine serum albumin in PBS for $1 \mathrm{~h}$, and further incubated overnight with the antibody of interest. The cells were washed extensively in PBS, followed by incubation with the appropriate secondary antibodies. ${ }^{35}$ DNA was visualized by adding DAPI at a concentration of $0.1 \mu \mathrm{g} / \mathrm{ml}$.

Western blotting. Cells were washed with ice-cold PBS, collected by scraping, and then lysed in lysis buffer $(50 \mathrm{mM}$ Tris pH 7.5, $100 \mathrm{mM} \mathrm{NaCl}, 1 \mathrm{mM}$ $\mathrm{Na}_{3} \mathrm{VO}_{4}, 50 \mathrm{mM} \mathrm{NaF}$, and $1 \%$ Triton $\mathrm{X}-100$ ). Cell lysates were subjected to SDS-PAGE and transferred to Immobilon P membranes (Millipore, Bllerica, MA, USA) ${ }^{36}$ Membranes were blocked for $2 \mathrm{~h}$ in TBST $(0.1 \%$ Tween $20,137 \mathrm{mM}$

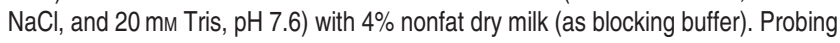
with the specific antibody was carried out for $2 \mathrm{~h}$ at room temperature in blocking buffer containing $0.02 \%$ sodium azide. After washing three times in TBST, the membranes were probed with $200 \mathrm{ng} / \mathrm{ml}$ peroxidase-labeled goat antirabbit IgG for $1 \mathrm{~h}$ in TBST. After extensive washing in TBST, the membranes were processed for enhanced chemiluminescence using ImmunoStar reagents (Wako). Western blots were imaged using a LAS-4000 luminescent image analyzer (Fuji, Tokyo, Japan).

Flow cytometry. siRNA-transfected cells were trypsinized, washed twice with PBS, and fixed with $70 \%$ ethanol at $-20^{\circ} \mathrm{C}$ overnight. Fixed cells were resuspended in PBS containing $10 \mu \mathrm{g} / \mathrm{ml}$ RNase A and $20 \mu \mathrm{g} / \mathrm{ml} \mathrm{PI}$. Cellular DNA content was analyzed using a FACSCalibur flow cytometer and the CellQuest software (Becton Dickinson, Tokyo, Japan). For each sample, at least 10000 cells were counted.

Live-cell imaging. siRNA-transfected HeLa cells were reseeded on $35 \mathrm{~mm}$ glass-bottom dishes. Before imaging, the culture medium was replaced with $\mathrm{CO}_{2}$-independent medium (Invitrogen) containing 10\% FBS. Cells were maintained at $37^{\circ} \mathrm{C}$ and imaged using a BIOREVO BZ-9000 inverted microscope. Time-lapse images were acquired at $3 \mathrm{~min}$ intervals.

\section{Conflict of Interest}

The authors declare no conflict of interest.

Acknowledgements. Antibodies against Bub1 and BubR1 were kindly provided by S Taylor at the University of Manchester. This work was supported in part by a Grant-in-Aid from the Ministry of Education, Culture, Sports, Science, and Technology of Japan.

1. Vitale I, Galluzzi L, Castedo M, Kroemer G. Mitotic catastrophe: a mechanism for avoiding genomic instability. Nat Rev Mol Cell Biol 2011; 12: 385-392.

2. Bucher N, Britten CD. G2 checkpoint abrogation and checkpoint kinase-1 targeting in the treatment of cancer. Br J Cancer 2008; 98: 523-528.

3. Harrington EA, Bebbington D, Moore J, Rasmussen RK, Ajose-Adeogun AO, Nakayama T et al. VX-680, a potent and selective small-molecule inhibitor of the Aurora kinases, suppresses tumor growth in vivo. Nat Med 2004; 10: 262-267.

4. Jordan MA, Wilson L. Microtubules as a target for anticancer drugs. Nat Rev Cancer 2004; 4: $253-265$

5. Castedo M, Perfettini JL, Roumier T, Andreau K, Medema R, Kroemer G. Cell death by mitotic catastrophe: a molecular definition. Oncogene 2004; 23: 2825-2837.

6. Vakifahmetoglu H, Olsson M, Tamm C, Heidari N, Orrenius S, Zhivotovsky B. DNA damage induces two distinct modes of cell death in ovarian carcinomas. Cell Death Differ 2008; 15: 555-566

7. Niikura Y, Dixit A, Scott R, Perkins G, Kitagawa K. BUB1 mediation of caspaseindependent mitotic death determines cell fate. J Cell Biol 2007; 178: 283-296.

8. Huang XF, Luo SK, Xu J, Li J, Xu DR, Wang LH et al. Aurora kinase inhibitory VX-680 increases Bax/Bcl-2 ratio and induces apoptosis in Aurora-A-high acute myeloid leukemia. Blood 2008; 111: 2854-2865.

9. Vogel C, Hager C, Bastians H. Mechanisms of mitotic cell death induced by chemotherapymediated G2 checkpoint abrogation. Cancer Res 2007; 67: 339-345.

10. Lüders J, Stearns T. Microtubule-organizing centres: a re-evaluation. Nat Rev Mol Cell Biol 2007; 8: 161-167.
11. Dammermann A, Merdes A. Assembly of centrosomal proteins and microtubule organization depends on PCM-1. J Cell Biol 2002; 159: 255-266.

12. Stillwell EE, Zhou J, Joshi HC. Human ninein is a centrosomal autoantigen recognized by CREST patient sera and plays a regulatory role in microtubule nucleation. Cell Cycle 2004; 3: $923-930$.

13. Kimura M, Kotani S, Hattori T, Sumi N, Yoshioka T, Todokoro K et al. Cell cycle-dependent expression and spindle pole localization of a novel human protein kinase, Aik, related to Aurora of Drosophila and yeast Ipl1. J Biol Chem 1997; 272: 13766-13771.

14. Cassimeris L, Morabito J. TOGp, the human homolog of XMAP215/Dis1, is required for centrosome integrity, spindle pole organization, and bipolar spindle assembly. Mol Biol Cell 2004; 15: 1580-1590.

15. Gergely F, Karlsson C, Still I, Cowell J, Kilmartin J, Raff JW. The TACC domain identifies a family of centrosomal proteins that can interact with microtubules. Proc Natl Acad Sci USA 2000; 97: 14352-14357.

16. Carmena M, Ruchaud S, Earnshaw WC. Making the Auroras glow: regulation of Aurora A and B kinase function by interacting proteins. Curr Opin Cell Biol 2009; 21: 796-805.

17. Hata T, Furukawa T, Sunamura M, Egawa S, Motoi F, Ohmura $N$ et al. RNA interference targeting aurora kinase a suppresses tumor growth and enhances the taxane chemosensitivity in human pancreatic cancer cells. Cancer Res 2005; 65: 2899-2905.

18. Schneider L, Essmann F, Kletke A, Rio P, Hanenberg H, Wetzel W et al. The transforming acidic coiled coil 3 protein is essential for spindle-dependent chromosome alignment and mitotic survival. J Biol Chem 2007; 282: 29273-29283.

19. Reinhardt HC, Schumacher B. The p53 network: cellular and systemic DNA damage responses in aging and cancer. Trends Genet 2012; 28: 128-136.

20. Debernardis D, Siré EG, De Feudis P, Vikhanskaya F, Valenti M, Russo P et al. p53 status does not affect sensitivity of human ovarian cancer cell lines to paclitaxel. Cancer Res 1997; 57: 870-874.

21. Toh WH, Nam SY, Sabapathy K. An essential role for p73 in regulating mitotic cell death. Cell Death Differ 2010; 17: 787-800.

22. Wu YC, Yen WY, Ho HY, Su TL, Yih LH. Glyfoline induces mitotic catastrophe and apoptosis in cancer cells. Int J Cancer 2010; 126: 1017-1028.

23. Antoni L, Sodha N, Collins I, Garrett MD. CHK2 kinase: cancer susceptibility and cancer therapy-two sides of the same coin? Nat Rev Cancer 2007; 7: 925-936.

24. Tsvetkov L, Xu X, Li J, Stern DF. Polo-like kinase 1 and Chk2 interact and co-localize to centrosomes and the midbody. J Biol Chem 2003; 278: 8468-8475.

25. Takada S, Kelkar A, Theurkauf WE. Drosophila checkpoint kinase 2 couples centrosome function and spindle assembly to genomic integrity. Cell 2003; 113: 87-99.

26. Hirota T, Kunitoku N, Sasayama T, Marumoto T, Zhang D, Nitta M et al. Aurora-A and an interacting activator, the LIM protein Ajuba, are required for mitotic commitment in human cells. Cell 2003; 114: 585-598.

27. Cheng TS, Hsiao YL, Lin CC, Hsu CM, Chang MS, Lee $\mathrm{Cl}$ et al. hNinein is required for targeting spindle-associated protein Astrin to the centrosome during the $\mathrm{S}$ and $\mathrm{G} 2$ phases. Exp Cell Res 2007; 313: 1710-1721.

28. Gergely F, Draviam VM, Raff JW. The ch-TOG/XMAP215 protein is essential for spindle pole organization in human somatic cells. Genes Dev 2003; 17: 336-341.

29. Bahe S, Stierhof YD, Wilkinson CJ, Leiss F, Nigg EA. Rootletin forms centriole-associated filaments and functions in centrosome cohesion. J Cell Biol 2005; 171: 27-33.

30. Lüders J, Patel UK, Stearns T. GCP-WD is a gamma-tubulin targeting factor required for centrosomal and chromatin-mediated microtubule nucleation. Nat Cell Biol 2006; 8: 137-147.

31. Johnson VL, Scott MI, Holt SV, Hussein D, Taylor SS. Bub1 is required for kinetochore localization of BubR1, Cenp-E, Cenp-F and Mad2, and chromosome congression. J Cell Sci 2004; 117: 1577-1589.

32. Sudo T, Nitta M, Saya H, Ueno NT. Dependence of paclitaxel sensitivity on a functional spindle assembly checkpoint. Cancer Res 2004; 64: 2502-2508.

33. Takagaki K, Katsuma S, Kaminishi Y, Horio T, Tanaka T, Ohgi T et al. Role of Chk1 and Chk2 in Ara-C-induced differentiation of human leukemia K562 cells. Genes Cells 2005; 10: $97-106$.

34. Taylor SS, Hussein D, Wang Y, Elderkin S, Morrow CJ. Kinetochore localization and phosphorylation of the mitotic checkpoint components Bub1 and BubR1 are differentially regulated by spindle events in human cells. J Cell Sci 2001; 114: 4385-4395.

35. Kimura M, Okano Y. Human Misato regulates mitochondrial distribution and morphology. Exp Cell Res 2007; 313: 1393-1404.

36. Kimura M, Matsuda Y, Yoshioka T, Okano Y. Cell cycle-dependent expression and centrosome localization of a third human aurora/lpl1-related protein kinase, AlK3. J Biol Chem 1999; 274: 7334-7340.

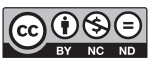

Cell Death and Disease is an open-access journal published by Nature Publishing Group. This work is licensed under a Creative Commons Attribution-NonCommercialNoDerivs 3.0 Unported License. To view a copy of this license, visit http://creativecommons.org/licenses/by-nc-nd/3.0/ 